\title{
A phase I study of selinexor in combination with high-dose cytarabine and mitoxantrone for remission induction in patients with acute myeloid leukemia
}

Amy Y. Wang ${ }^{1}$, Howard Weiner ${ }^{2}$, Margaret Green², Hua Chang ${ }^{3}$, Noreen Fulton ${ }^{2}$, Richard A. Larson², Olatoyosi Odenike², Andrew S. Artz², Michael R. Bishop², Lucy A. Godley², Michael J. Thirman², Satyajit Kosuri², Jane E. Churpek ${ }^{2}$, Emily Curran ${ }^{2}$, Kristen Pettit ${ }^{4}$, Wendy Stock ${ }^{2}$ and Hongtao Liu ${ }^{2 *}$

\begin{abstract}
Background: Novel therapies for patients with acute myeloid leukemia (AML) are imperative, particularly for those with high-risk features. Selinexor, an exportin 1 (XPO1/CRM1) inhibitor, has demonstrated anti-leukemia activity as a single agent, as well as in combination with anthracyclines and/or DNA-damaging agents.

Methods: We report the findings of a phase I dose escalation trial with cohort expansion in 20 patients with newly diagnosed or relapsed/refractory AML that combined selinexor with age-adjusted high-dose cytarabine and mitoxantrone (HiDAC/Mito).

Results: Three (15\%) patients received the initial dose of $60 \mathrm{mg}$ of selinexor $\left(\sim 35 \mathrm{mg} / \mathrm{m}^{2}\right)$, and $17(85 \%)$ received the target level of $80 \mathrm{mg}\left(\sim 50 \mathrm{mg} / \mathrm{m}^{2}\right)$. No dose-limiting toxicities were observed. Common adverse events included febrile neutropenia (70\%), diarrhea (40\%), anorexia (30\%), electrolyte abnormalities (30\%), bacteremia (25\%) , cardiac toxicities (25\%), fatigue (25\%), and nausea/vomiting (25\%). None were unexpected given the HiDAC/Mito regimen. Serious adverse events occurred in 6 (30\%) patients; one was fatal. Ten (50\%) patients achieved a complete remission (CR), 3 (15\%) achieved CR with incomplete recovery (CRi), 1 (5\%) achieved partial remission (PR), and 6 (30\%) had progressive disease for an overall response rate (ORR) of 70\%. Eight of 14 (57\%) responders proceeded to allogeneic stem cell transplantation. Correlative studies of WT1 levels showed persistently detectable levels in patients who either did not respond or relapsed quickly after induction.
\end{abstract}

Conclusion: The selinexor/HiDAC/Mito regimen is feasible and tolerable at selinexor doses of $80 \mathrm{mg} /$ day $(\sim 50 \mathrm{mg} /$ $\mathrm{m}^{2} /$ day) twice weekly. The recommended phase II dose is $80 \mathrm{mg}$ and warrants further study in this combination.

Trial registration: ClinicalTrials.gov, NCT02573363. Registered October 5, 2015

Keywords: AML, Selinexor, Induction chemotherapy, XPO1/CRM1

\footnotetext{
* Correspondence: hliu2@medicine.bsd.uchicago.edu

${ }^{2}$ Department of Medicine, Section of Hematology/Oncology, University of

Chicago Medicine, 5841 S. Maryland, MC 2115, Chicago, IL 60637-1470, USA

Full list of author information is available at the end of the article
} 


\section{Background}

Acute myeloid leukemia (AML) is the most common acute leukemia in adults and is characterized by a poor prognosis with a 5 -year survival rate of approximately $30 \%$ [1]. Even patients who achieve remission to initial therapy often relapse, and certain subgroups such as those with therapyrelated AML, older patients, and those with relapsed or refractory disease have a particularly poor outcome $[2,3]$. Novel agents that are active and able to be widely applied are imperative. Selinexor (KPT-330), an exportin 1 (XPO1) inhibitor, may be such an agent.

$\mathrm{XPO} 1$ is the exclusive, nuclear exporter of most major tumor suppressor proteins (TSP) and growth regulatory proteins (GRP), including p53, p21, p73, FOXO1, and NPM1 [4-6]. In leukemia and solid tumors, XPO1 is overexpressed leading to enhanced transport of these proteins to the cytoplasm, thereby neutralizing their anti-neoplastic functions and functionally inactivating TSP/GRP. Elevated levels of XPO1 have been independently associated with a worse prognosis in adults with AML $[7,8]$. These findings presented an attractive target for the development of a novel class of XPO1 inhibitors. However, the earliest compounds of XPO1 inhibitors had faced significant toxicities in phase I clinical trials requiring discontinuation, while others were never studied in a clinical setting [9].

Selinexor (KPT-330) is an oral, first-in-class, slowly reversible, and potent agent that was among the next group of selective inhibitor of nuclear export (SINE) compounds to be developed, which also included KPT335, KPT-185, KPT-276, and KPT-251, many of which have since been studied in clinical trials. The SINE compounds bind to residue Cys528 of XPO1 and blocks the transport of cargo proteins [5]. In both AML primary samples and murine xenograft models, SINE compounds have been shown to reduce XPO1 levels and enhance the nuclear accumulation of p53 [10,11]. A phase I clinical trial of selinexor in patients with advanced AML demonstrated it to have a manageable safety profile at $35 \mathrm{mg} / \mathrm{m}^{2}(60 \mathrm{mg})$ and to be efficacious in a single-agent setting [12].

In vivo and in vitro evidence suggest synergistic activity against leukemic cells by combining selinexor with anthracyclines or DNA-damaging agents [13]. The combination of high-dose cytarabine (HiDAC) with the anthracycline mitoxantrone (Mito) is an effective induction regimen for patients with AML [14-17] and is frequently utilized at the University of Chicago as frontline therapy for patients with high-risk AML, either de novo or relapsed/refractory. The HiDAC/Mito regimen demonstrated an overall response rate (ORR) of $55 \%$ in the high-risk AML population at this institution [16] and an ORR of $82 \%$ in another study of previously untreated patients with therapyrelated myeloid neoplasms [17].
Based on phase I safety and efficacy data of selinexor in patients with AML, the promising results with HiDAC/Mito induction, and the in vivo and in vitro data suggesting synergistic killing of AML cell lines by combining selinexor and anthracyclines, we hypothesized that selinexor would sensitize AML cells to the cytotoxic effects of HiDAC/Mito. We monitored the impact on minimal residual disease (MRD) by tracking the level of Wilms tumor 1 (WT1) expression. WT1 levels in the peripheral blood predict relapse after remission, and their levels after consolidation therapy are closely correlated with survival and early relapse $[18,19]$. In this phase I study, we describe the safety, tolerability, correlative molecular studies, and activity of selinexor in combination with $\mathrm{HiDAC} / \mathrm{Mito}$ for remission induction in patients with previously untreated or relapsed/refractory $(\mathrm{R} / \mathrm{R}) \mathrm{AML}$

\section{Methods}

\section{Study subjects and design}

We performed a phase I dose escalation trial with cohort expansion that combined increasing doses of selinexor with age-adjusted HiDAC/Mito (NCT02573363). The trial was approved by the Institutional Review Board at The University of Chicago (IRB15-0412). Patients with newly diagnosed or R/R AML, except acute promyelocytic leukemia, were eligible to enroll if they had adequate performance status (ECOG $\leq 2$ ), cardiac function (ejection fraction $>50 \%$ ), renal function (creatinine clearance $>30 \mathrm{~cm}^{3} / \mathrm{min}$ ), and hepatic function (transaminases $\leq 3.0$ times upper limit of normal). Exclusion criteria included active central nervous system (CNS) leukemia, pregnancy, recent treatment with any investigational agent, recent major surgery, concurrent malignancy under active treatment, active infection, or recent seizure or stroke. Dose escalation of selinexor was performed according to a $3+3$ design [20]. Patient enrollment began in October 2015.

\section{Treatment regimen}

Figure 1 shows the study schematic. HiDAC $\left(3 \mathrm{~g} / \mathrm{m}^{2}\right.$, or $2 \mathrm{~g} / \mathrm{m}^{2}$ if $>70$ years, intravenously over $4 \mathrm{~h}$ ) followed immediately by Mito $\left(30 \mathrm{mg} / \mathrm{m}^{2}\right.$, or $20 \mathrm{mg} / \mathrm{m}^{2}$ if $>70$ years, intravenously over $1 \mathrm{~h}$ ) were administered on days 1 and 5. This regimen was based on several studies demonstrating efficacy in response and leukemia cell dynamics $[14,15]$. Selinexor was given orally on days $2,4,9$, and 11. Initial selinexor dose was $60 \mathrm{mg}\left(\sim 35 \mathrm{mg} / \mathrm{m}^{2}\right.$ for an average adult) followed by dose escalation to a target level of $80 \mathrm{mg}\left(\sim 50 \mathrm{mg} / \mathrm{m}^{2}\right)$. Patients who failed to achieve remission after induction were taken off the study. A second cycle of induction was not given. Patients who entered remission proceeded to allogeneic hematopoietic cell transplantation (HCT), if feasible, or 


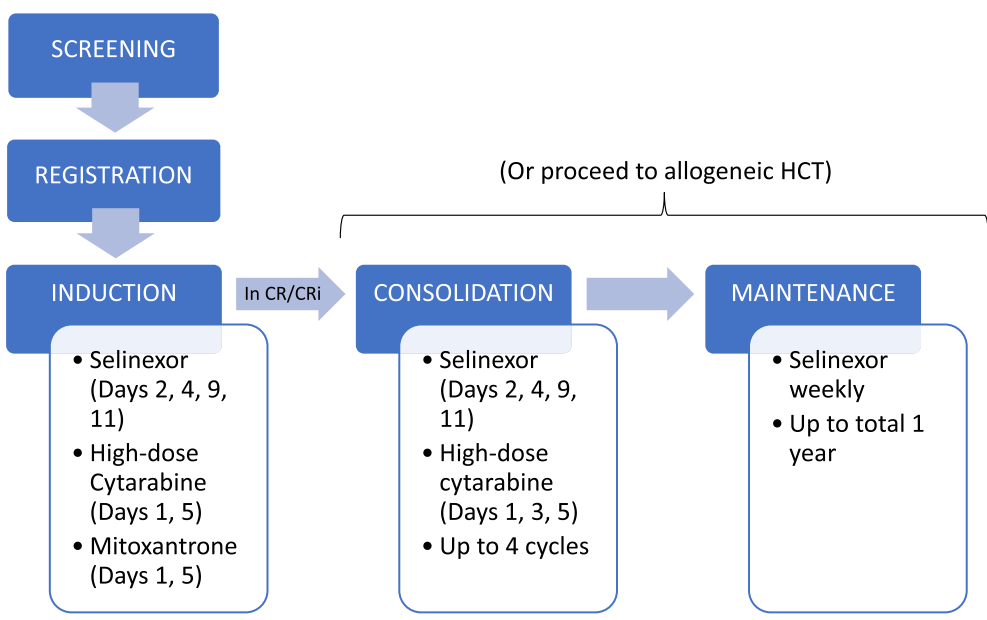

Fig. 1 Study schematic. Schematic of phase 1 dose escalation study. Selinexor dosing remained unchanged for all phases, except for 2 patients who received reduced selinexor dosing during consolidation. Twenty patients entered induction, and 14 achieved a response. No patients received a second cycle of induction. No patients withdrew. Six patients entered consolidation, but 1 soon proceeded to allogeneic HCT. Ultimately, 8 underwent allogeneic HCT. One patient quickly relapsed after induction prior to starting the next phase of therapy. $\mathrm{HCT}=$ hematopoietic cell transplantation

consolidation chemotherapy with HiDAC and selinexor for up to four cycles at the same dose, followed by maintenance therapy with weekly selinexor for up to 1 year. Dose adjustments were allowed during consolidation or maintenance if patients experienced grade $>3$ toxicities. The selinexor doses and schedule were determined based on results from prior phase I studies in hematologic malignancies (KCP-330-001 [NCT01607892]), solid tumors (KCP-330-002 [NCT01607905]), and sarcomas (KCP-330003 [NCT01896505]). These studies demonstrated a maximum tolerated dose (MTD) of $80-120 \mathrm{mg}$ given twice weekly over a 4 -week cycle but recommended a lower dose at 60-80 mg to better enable successful chronic use. Prophylactic antimicrobial and anti-emetic agents were administered according to institutional guidelines.

\section{Toxicity assessment}

Patients in the dose escalation cohorts were monitored for dose-limiting toxicities (DLT) for up to 56 days. DLT was defined as any grade $\geq 3$ treatment-related non-hematologic toxicity, or persistent bone marrow aplasia in the absence of AML, or any grade 4 neutropenia or thrombocytopenia at day 56 of induction therapy not explained by disease recurrence or infection. Once a dose level was declared tolerable, more patients could be enrolled at that level to provide additional safety, tolerability, and efficacy data. Patients in the dose expansion cohort were closely monitored for adverse events (AE) but not assessed for DLT per the study design. All AEs were noted, including any serious adverse events (SAE), which were AEs that resulted in death, were lifethreatening (patient is at immediate risk of death from the event as it occurred), required inpatient hospitalization or prolongation of existing hospitalization for $\geq 24 \mathrm{~h}$, resulted in significant disability/incapacity, or resulted in a congenital anomaly or birth defect.

\section{Response criteria and monitoring}

The primary objective was to determine the recommended phase II dose (RP2D) of selinexor with HiDAC/Mito. Secondary objectives were to determine the complete remission (CR) rate, toxicities, progression-free survival (PFS) and overall survival (OS) rates, and allogeneic HCT success rate, defined as the number of patients who proceeded to HCT following remission. CR was defined as being transfusion independent with an absolute neutrophil count (ANC) $>1.0 \times 10^{9} / \mathrm{L}$, platelet count $>100 \times 10^{9} / \mathrm{L}$, and bone marrow blasts $<5 \%[21,22]$. CR with incomplete recovery (CRi) was defined as meeting all CR criteria except for residual neutropenia $\left(<1.0 \times 10^{9} / \mathrm{L}\right)$ or thrombocytopenia $\left(<100 \times 10^{9} / \mathrm{L}\right)$. Partial remission $(\mathrm{PR})$ was defined as a decrease in pre-treatment bone marrow blast percentage by at least $50 \%$ and to within the range of $5-25 \%$, while otherwise meeting all hematologic criteria of CR. Lastly, a treatment failure (TF) was defined as resistant disease, relapse, or death [22]. All patients were followed until disease progression, withdrawal, death, or up to 1 year after completion of all protocol treatment.

\section{Correlative molecular studies}

To assess WT1 levels as a marker of MRD, peripheral blood and marrow aspirate samples were collected before induction, at day 12 , at the patient's blood count recovery or prior to day 56 , and again at relapse. Bone marrow aspirate and/or peripheral blood mononuclear cell cDNA samples and template controls were assayed by quantitative real-time PCR (qRT-PCR) analysis utilizing Taqman gene expression assays (Life Technologies) 
for WT1 (Hs01103751) and ABL (Hs01104728) in triplicate using LightCycler 48011 (Roche). All transcript expression levels were determined by reference to standard curves generated from fivefold serial dilutions of $\mathrm{K} 562$ cell line cDNA (0.08-250 ng). The absolute transcript copy number was normalized to the endogenous control gene, $A B L 1$.

To demonstrate the effect of selinexor with HiDAC/ Mito on the bone marrow, routine hematoxylin and eosin $(\mathrm{H} \& \mathrm{E})$ and immunohistochemistry $(\mathrm{IHC})$ staining for Ki67, p53, SMAD4, Rb, and p21 were performed with formalin-fixed paraffin-embedded sections cut at $5 \mu \mathrm{m}$. For IHC staining, slides were baked at $65{ }^{\circ} \mathrm{C}$ for $30 \mathrm{~min}$, processed for deparaffinization and rehydration, and then placed in Declare working buffer, steamcooked for antigen retrieval, cooled, and transferred to $3 \%$ hydrogen peroxide to block endogenous hydrogenase activity. Protein block was applied before primary antibodies were incubated with slides. Cell Marque Hi-Def Polymer Amplifier and Secondary Antibody were applied sequentially at room temperature as per manufacturer's instructions. DAB chromogen was used for color reaction. Slides were counterstained with hematoxylin, dehydrated, mounted, and cover-slipped. IHC staining was performed on a Biogenex I6000 automated stainer. Digital images of the slides were obtained through an Aperio AT Turbo image scanner at $\times 20$.

\section{Statistics}

Survival rates were calculated using the Kaplan-Meier method in the statistical software R (version).

\section{Results}

\section{Patient characteristics}

Twenty patients enrolled and received selinexor with HiDAC/Mito. Table 1 lists the patient characteristics. Three patients received selinexor $60 \mathrm{mg}$, and 17 patients received $80 \mathrm{mg}$. Fourteen (70\%) patients were female. The median age was 61 with a range of 44-75 years. Twelve (60\%) patients had untreated AML, and $8(40 \%)$ patients had R/R disease. Twelve (60\%) patients were diagnosed with de novo AML, and 8 (40\%) patients had AML after a myelodysplastic syndrome (MDS). There were no patients with therapy-related AML. Four (20\%) patients had favorable risk, $8(40 \%)$ had intermediate risk, and $8(40 \%)$ had adverse risk AML by European LeukemiaNet criteria. Five (25\%) patients had FLT3 mutations; 3 were internal tandem duplications (ITD), and 2 were point mutations in the tyrosine kinase domain (TKD). Two (10\%) patients had CEBPA mutations, and 5 (25\%) patients had NPM1 mutations. The median number of prior regimens for the $R / R$ patients was 2 and included cytarabine with anthracycline $(7+3)$, HiDAC, hypomethylating agents, tyrosine
Table 1 Characteristics of the patients

\begin{tabular}{|c|c|}
\hline Patient characteristics & Number (\%) \\
\hline Total patients enrolled & 20 \\
\hline Selinexor 60 mg & 3 \\
\hline Selinexor 80 mg & 17 \\
\hline Female & $14(70 \%)$ \\
\hline Median age (years, range) & $61(44-76)$ \\
\hline \multicolumn{2}{|l|}{ Disease state on enrollment } \\
\hline Untreated AML & $12(60 \%)$ \\
\hline Relapsed or refractory $\mathrm{AML}^{\mathrm{a}}$ & $8(40 \%)$ \\
\hline \multicolumn{2}{|l|}{ Initial AML diagnosis } \\
\hline De novo AML & $12(60 \%)$ \\
\hline Secondary AML after MDS & $8(40 \%)$ \\
\hline \multicolumn{2}{|c|}{ European LeukemiaNet genetic risk group } \\
\hline Favorable & $4(20 \%)$ \\
\hline Intermediate I/II & $8(40 \%)$ \\
\hline Adverse & $8(40 \%)$ \\
\hline \multicolumn{2}{|l|}{ Acquired mutation status } \\
\hline FLT3 & $\begin{array}{l}3(15 \%) \text { with ITD, } 2(10 \%) \\
\text { with TKD mutation }\end{array}$ \\
\hline CEBPA & $\begin{array}{l}2(10 \%) \text { (one had bi-allelic } \\
\text { mutation) }\end{array}$ \\
\hline NPM1 & 5 (25\%) (3 with FLT3 mutation) \\
\hline $\begin{array}{l}\text { Median number of prior regimens } \\
(\mathrm{R} / \mathrm{R} \text { only })^{\mathrm{a}}\end{array}$ & 2 (range, 1-3) \\
\hline
\end{tabular}

kinase inhibitors, FLAG-IDA, ATRA (for EV1 translocation), and other investigational agents.

\section{Toxicity}

Myelosuppression was the most common hematologic toxicity and was universal. However, patients had longer than expected duration of neutropenia and thrombocytopenia. The median time to achieve CR was 37.5 days (range 26-50 days). The median time to achieve an ANC > $0.5 \times 10^{9} / \mathrm{L}$ was 31 days (range $22-48$ days) and platelet count $>20 \times 10^{9} / \mathrm{L}$ was 25 days (range $19-38$ days).

Table 2 lists the non-hematologic adverse events observed during induction, consolidation, and maintenance phases, of which febrile neutropenia was the most common at $70 \%$. No patients required intensive care because of infection. Gastrointestinal toxicities were common: diarrhea (40\%), anorexia (30\%), and nausea and vomiting (25\%). Most were grade 1 or 2 and manageable with supportive therapies. Other frequent adverse events included electrolyte abnormalities (hyponatremia or hypokalemia in $30 \%)$, bacteremia (25\%), cardiac toxicities $(25 \%)$, fatigue (25\%), pneumonia $(20 \%)$, and 
Table 2 Adverse events observed in > 5\% of patients

\begin{tabular}{|c|c|c|c|c|c|}
\hline Adverse events & $\begin{array}{l}\text { Total, } \\
n(\%)\end{array}$ & $\begin{array}{l}\text { Grades } 1 \\
\text { and } 2\end{array}$ & Grade 3 & Grade 4 & Grade 5 \\
\hline Febrile neutropenia & $14(70 \%)$ & & 14 & & \\
\hline Diarrhea & $8(40 \%)$ & 8 & & & \\
\hline Anorexia & $6(30 \%)$ & 6 & & & \\
\hline $\begin{array}{l}\text { Electrolyte } \\
\text { abnormalities }\end{array}$ & $6(30 \%)$ & 6 & & & \\
\hline Bacteremia & $5(25 \%)$ & & 5 & & \\
\hline Cardiac toxicity $^{b}$ & $5(25 \%)$ & 2 & 3 & & \\
\hline Nausea/vomiting & $5(25 \%)$ & 4 & $1^{\mathrm{a}}$ & & \\
\hline Fatigue & $5(25 \%)$ & 5 & & & \\
\hline Pneumonia & $4(20 \%)$ & & 4 & & \\
\hline Alopecia & $4(20 \%)$ & 4 & & & \\
\hline $\begin{array}{l}\text { Line-associated } \\
\text { DVT }\end{array}$ & $3(15 \%)$ & & 3 & & \\
\hline Acute kidney injury & $3(15 \%)$ & 3 & & & \\
\hline Rash & $3(15 \%)$ & 3 & & & \\
\hline Mood disorders & $3(15 \%)$ & 3 & & & \\
\hline $\begin{array}{l}\text { Clostridium difficile } \\
\text { colitis }\end{array}$ & $2(10 \%)$ & 2 & & & \\
\hline $\begin{array}{l}\text { Syncope/pre- } \\
\text { syncope }\end{array}$ & $2(10 \%)$ & 1 & 1 & & \\
\hline $\begin{array}{l}\text { Upper respiratory } \\
\text { infection }\end{array}$ & $2(10 \%)$ & 2 & & & \\
\hline Mucositis & $2(10 \%)$ & 2 & & & \\
\hline Transaminitis & $2(10 \%)$ & 2 & & & \\
\hline Psychosis & $2(10 \%)$ & 2 & & & \\
\hline Typhlitis & $1(5 \%)$ & & 1 & & \\
\hline Hypoxia & $1(5 \%)$ & & 1 & & \\
\hline $\begin{array}{l}\text { Urinary tract } \\
\text { infection }\end{array}$ & $1(5 \%)$ & & $1^{\mathrm{a}}$ & & \\
\hline Cerebellar toxicity & $1(5 \%)$ & & $1^{a}$ & & \\
\hline Hemorrhagic stroke & $1(5 \%)$ & & & & $1^{\mathrm{a}}$ \\
\hline Cellulitis & $1(5 \%)$ & & $1^{\mathrm{a}}$ & & \\
\hline Endocarditis & $1(5 \%)$ & & $1^{a}$ & & \\
\hline \multirow[t]{2}{*}{ Total } & 93 & 55 & 37 & 0 & 1 \\
\hline & \multicolumn{5}{|c|}{$\begin{array}{l}\text { Adverse events occurring } \leq 5 \% \text { not listed above: } \\
\text { diverticulitis, edema, dysuria, musculoskeletal pain, } \\
\text { vaginitis, plantar fasciitis, dry mouth, dysphagia, } \\
\text { otitis externa, conjunctivitis, gingivitis, chest pain, } \\
\text { hyperbilirubinemia, hypoalbuminemia, INR increased, } \\
\text { peripheral neuropathy, and insomnia. }\end{array}$} \\
\hline
\end{tabular}

DVT deep vein thrombosis; INR international normalized ratio ${ }^{\text {a }}$ Serious adverse event

${ }^{\mathrm{b} C a r d i a c}$ toxicities included reduction in ejection fraction (2), atrial fibrillation (1), sinus bradycardia (1), and prolonged QT interval (2)

alopecia (20\%). Cardiac toxicities included 2 patients with reduced ejection fraction (1 had prior history of anthracycline exposure), 1 with atrial fibrillation, 1 with right bundle branch block, and 1 with prolonged QT interval due to anti-emetics. SAEs occurred in 6
(30\%) patients, which included a severe urinary tract infection (during maintenance), cerebellar toxicity, hemorrhagic stroke, cellulitis (during consolidation), endocarditis, and intractable nausea/vomiting.

No DLT was observed during the dose escalation phase. However, 3 patients in the subsequent expansion cohort had noteworthy toxicities: one patient with a hypocellular bone marrow prior to induction had an aplastic marrow lasting beyond day 56; another suffered a hemorrhagic stroke with platelet count of $4000 / \mu \mathrm{L}$ and died during induction; a third patient required total parenteral nutrition (TPN) briefly due to severe nausea, vomiting, and anorexia, despite supportive therapies.

\section{Response}

Of the 20 patients evaluable for response, 10 (50\%) achieved CR, 3 (15\%) achieved CRi, 1 (5\%) achieved PR, and 6 (30\%) had TF (Table 3). The patient who achieved PR received selinexor $60 \mathrm{mg}$ monotherapy twice a week for 2 weeks through an approved protocol amendment and achieved $\mathrm{CRi}$, which allowed the patient to proceed to a HCT. The overall response rate (ORR) was $70 \%(n=14 / 20)$. Patients who received selinexor $80 \mathrm{mg}(n=17)$ had an ORR of $76 \%$; the ORR was $33 \%$ for patients who received $60 \mathrm{mg}(n=3)$. The ORR was $80 \%$ for younger patients (age $\leq 60$ ) and $50 \%$ in older patients. Patients with newly diagnosed AML had an ORR of $92 \%$ (vs 38\% for R/R). All patients with favorable risk achieved $\mathrm{CR}$, whereas the intermediate and adverse risk groups achieved an ORR of 67 and 63\%, respectively. Statistical significance was not calculated due to small sample size.

Nineteen (95\%) patients completed induction therapy. One patient $(5 \%)$ died during induction. One patient who failed to respond to initial induction transferred care to another institution to enroll in another clinical trial. No patients withdrew from the study due to AEs. Of the 14 patients who responded, 5 proceeded to consolidation, 8 underwent allogeneic HCT (of which 1 was initially in consolidation prior to transplant), and 1 relapsed soon after achieving $\mathrm{CRi}$ and enrolled in another clinical trial. No dose reductions were needed during induction, but two patients required a reduction of selinexor from $80 \mathrm{mg}$ to $60 \mathrm{mg}$ during cycle 1 and cycle 2 of consolidation due to fatigue and nausea. The number of patients undergoing allogeneic HCT was $40 \%$ $(n=8 / 20)$ for all patients and $57 \%(n=8 / 14)$ for responding patients.

Of note, 5 (25\%) patients had FLT3-mutated AML (3 had ITD and 2 had TKD mutations) (Table 1). Four of the $5(80 \%)$ achieved a CR. Only one patient had received a prior FLT3 inhibitor on another clinical trial. All 5 patients went on to allogeneic HCT. Five (25\%) patients had NPM1 mutations, of which 3 
Table 3 Responses to the treatment

\begin{tabular}{|c|c|c|c|c|c|c|}
\hline Response rates & Total & CR (\%) & CRi (\%) & PR (\%) & TF $(\%)$ & ORR (\%) \\
\hline \multicolumn{7}{|l|}{ Dose } \\
\hline $60 \mathrm{mg}$ & $3(15 \%)$ & $1(33 \%)$ & 0 & 0 & $2(66 \%)$ & $1(33 \%)$ \\
\hline 80 mg & 17 (85\%) & $9(53 \%)$ & $3(18 \%)$ & $1(6 \%)$ & $4(24 \%)$ & $13(76 \%)$ \\
\hline \multicolumn{7}{|l|}{ Age } \\
\hline Age $\leq 60^{a}$ & $10(50 \%)$ & $6(60 \%)$ & $1(10 \%)$ & $1(10 \%)$ & $2(20 \%)$ & $8(80 \%)$ \\
\hline Age $>60^{\mathrm{b}}$ & $10(50 \%)$ & $4(40 \%)$ & $2(20 \%)$ & 0 & $4(40 \%)$ & $6(60 \%)$ \\
\hline \multicolumn{7}{|l|}{ AML diagnosis } \\
\hline Newly diagnosed & $12(60 \%)$ & $7(58 \%)$ & $3(25 \%)$ & $1(8 \%)$ & $1(8 \%)$ & $11(92 \%)$ \\
\hline Relapsed/refractory & $8(40 \%)$ & $3(38 \%)$ & 0 & 0 & $5(63 \%)$ & $3(38 \%)$ \\
\hline \multicolumn{7}{|c|}{ European LeukemiaNet risk group } \\
\hline Favorable & $3(15 \%)$ & $3(100 \%)$ & 0 & 0 & 0 & $3(100 \%)$ \\
\hline Intermediate |/II & $9(45 \%)$ & $4(44 \%)$ & $2(22 \%)$ & 0 & $3(33 \%)$ & $6(67 \%)$ \\
\hline Adverse & $8(40 \%)$ & $3(38 \%)$ & $1(13 \%)$ & $1(13 \%)$ & $3(38 \%)$ & $5(63 \%)$ \\
\hline Total & 20 (100\%) & $10(50 \%)$ & $3(15 \%)$ & $1(5 \%)$ & $6(30 \%)$ & $14(70 \%)$ \\
\hline
\end{tabular}

7 newly diagnosed; 3 relapsed/refractory

${ }^{5} 5$ newly diagnosed; 5 relapsed/refractory

also had a FLT3 mutation; all 5 patients achieved CR. While these numbers are small, further study of the effect of selinexor/HiDAC/Mito on the FLT3 mutation may prove fruitful [23].

\section{Survival}

At the time of this analysis, $80 \%(n=16 / 20)$ of patients were alive and the median survival has not been reached. The median follow-up time for all patients was 6.0 months (range, 8 days to $>14$ months), and one-year survival was projected at $69 \%$ (Fig. 2a). Of the 4 total deaths, 1 died during induction (on day 8 ), 2 patients who did not respond died from disease progression, and 1 responded but died after relapse. The projected 1-year progression-free survival was $68 \%$ (Fig. 2b).

\section{Correlative molecular studies}

WT1 levels were assessed as a marker of MRD by the serial collection of peripheral blood and marrow aspirates at baseline, at the day 12 nadir time point during induction, at count recovery or prior to day 56 , and at relapse. WT1 levels for 8 patients are summarized in Fig. 3. All patients showed a reduction in WT1 by day 12. In the 7 patients who responded to induction treatment and had samples analyzed, WT1 level was detectable at baseline (median normalized WT1 ratio $=0.200$ ), undetectable at day 12 (median $=0.000$ ), and either
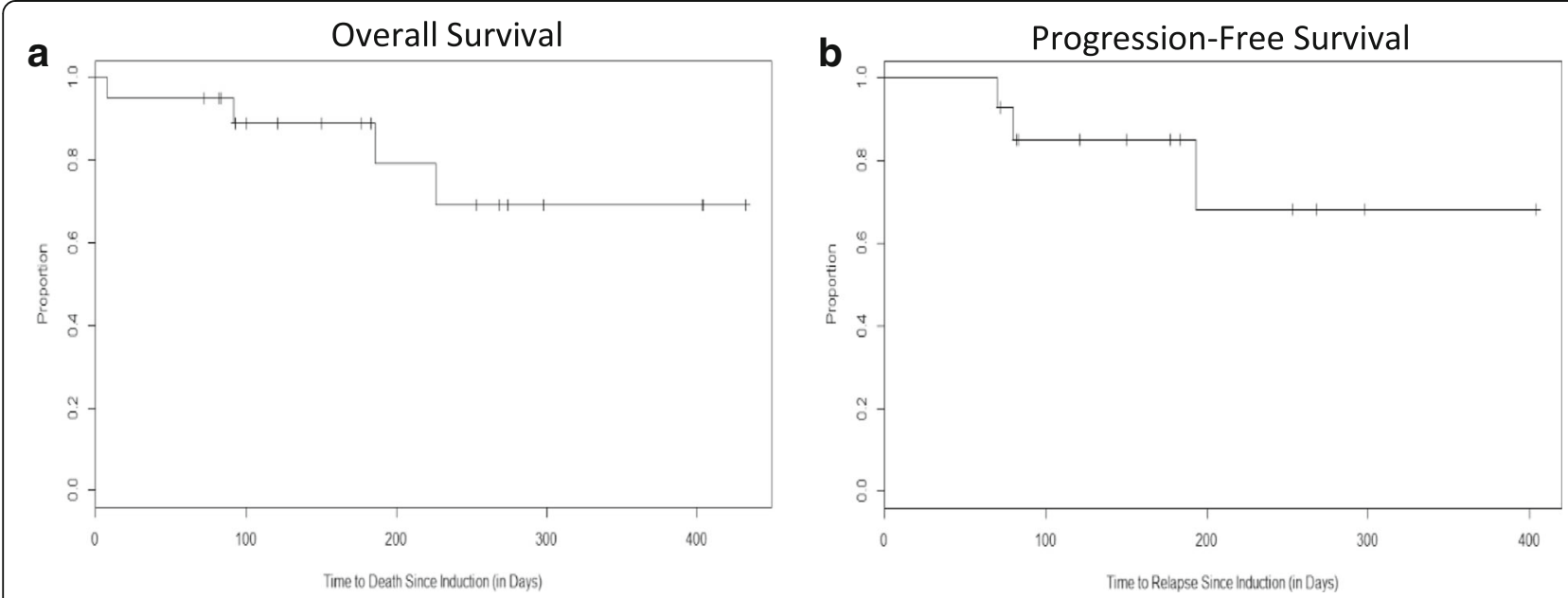

Fig. 2 Kaplan-Meier curves depicting patient survival and relapse since the start of induction. a Overall patient survival $(n=20)$. b Relapse-free survival $(n=14)$ 

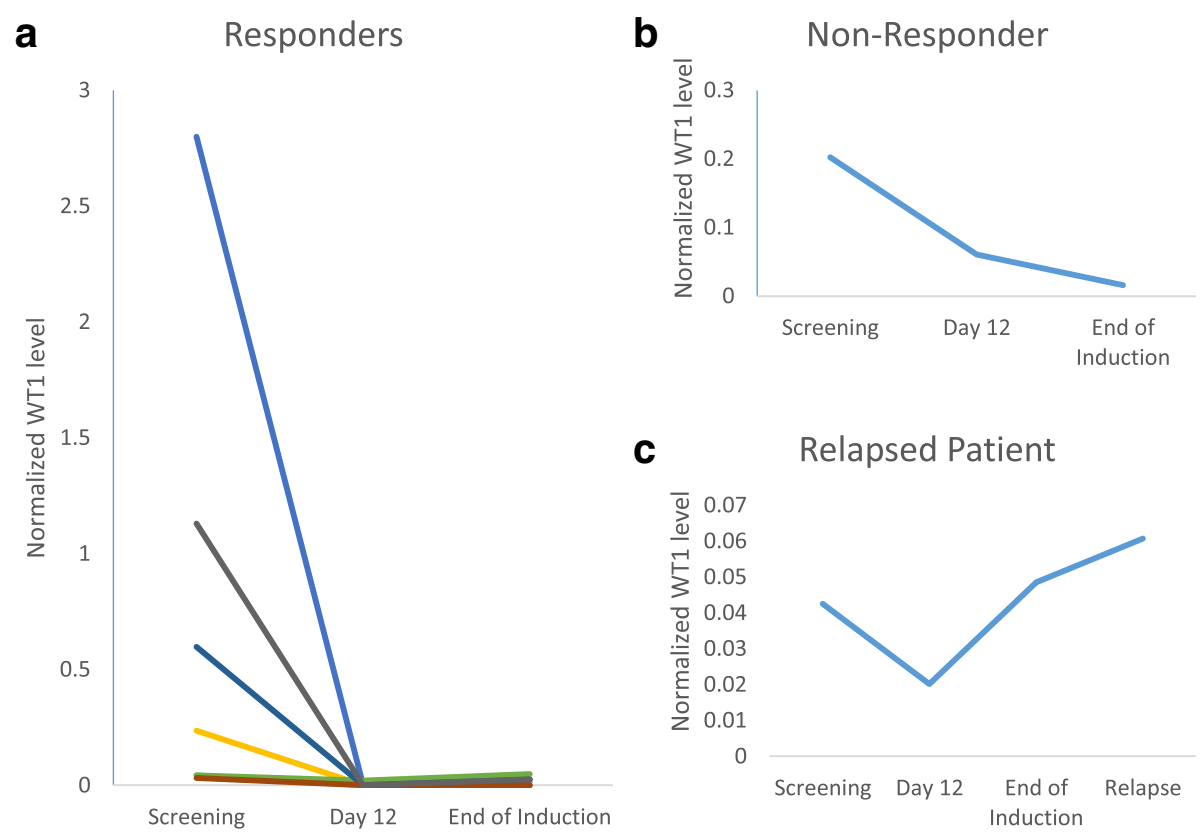

Fig. 3 WT1 levels at set time points in induction and at relapse. WT1 levels were assessed in bone marrow samples from 8 patients (7 CR/CRi/PR, 1 TF) shown here at the start of induction, at the day 12 nadir, and the end of induction defined as either count recovery or day 56, and at relapse if applicable. WT1 levels were normalized against the control ABL level. a WT1 levels of 7 patients who achieved a response. Screening baseline range 0.03-2.80; day 12 range 0.00-0.02; end of induction range 0.00-0.05. b One patient did not respond and maintained detectable WT1 at all time points $(0.20,0.06,0.02)$. c One of the 7 responders displayed in $\mathbf{a}$ initially achieved an incomplete response but then relapsed. This patient had detectable WT1 at all time points $(0.04,0.02,0.05,0.06$ at relapse)

remained undetectable or increased very slightly (median $=0.015)$ in the marrow at count recovery. One of the 8 patients failed to respond and retained a detectable level of WT1 at day 12 (Fig. 3b). Figure 3c highlights one patient who initially responded (achieved CRi) then relapsed; this patient also retained a detectable level of WT1 at day 12, then had an increase at the count recovery marrow, and subsequently a sharp increase of WT1 at the relapse marrow.

To demonstrate the activity of HiDAC/Mito and selinexor, IHC staining of DNA damage response proteins was performed on core biopsy slides at baseline, at the day 12 nadir, and at the count recovery marrow or day 56. Marked reductions in the proliferation marker Ki67 [24] were observed in the remaining tumor cells on the post-treatment biopsy slides of one patient who achieved $\mathrm{CR}$ and one who failed to respond (Fig. 4a). Staining for major TSPs p53, SMAD4, Rb, and p21 showed very little nuclear staining in the pre-treatment sample with an increase in nuclear staining intensity in the post-treatment marrow biopsy slides (Fig. 4b). This is consistent with the known effect of selinexor on tumor cells [10].

\section{Discussion}

The combination of selinexor with HiDAC/Mito for induction chemotherapy in AML is a feasible, tolerable, and effective regimen. This study resulted in an ORR of
$70 \%$ and a low induction death rate of $5 \%$. This phase I study demonstrates that this first-in-class SINE compound has an acceptable toxicity profile at the tested doses of 60 and $80 \mathrm{mg}$. The MTD was not determined, and no DLT was observed in the dose escalation phase. However, a frequent hematologic toxicity was prolonged thrombocytopenia and delayed neutrophil recovery. Four patients received filgrastim to promote count recovery, but this did not shorten count recovery time. Moreover, studies on filgrastim have not demonstrated a consistent benefit with respect to lower rates of infections and improved survival $[25,26]$. While patients eventually recovered blood counts, one patient with secondary AML had a hypocellular bone marrow prior to induction therapy and hence failed to recover blood counts by day 56 . Prior studies of selinexor alone have not reported any adverse effects on hematopoietic function [7, 12, 27]. Nevertheless, based on this experience, patients with a hypocellular marrow prior to induction should receive this regimen with particular caution, and dose-reduction of selinexor should be considered based on marrow cellularity and age.

Commonly reported non-infectious toxicities included diarrhea, anorexia, electrolyte abnormalities, nausea, vomiting, and fatigue, consistent with prior selinexor studies [12, 27-29]. The Larson et al. study [16] of HiDAC/Mito alone in high-risk AML patients offered 


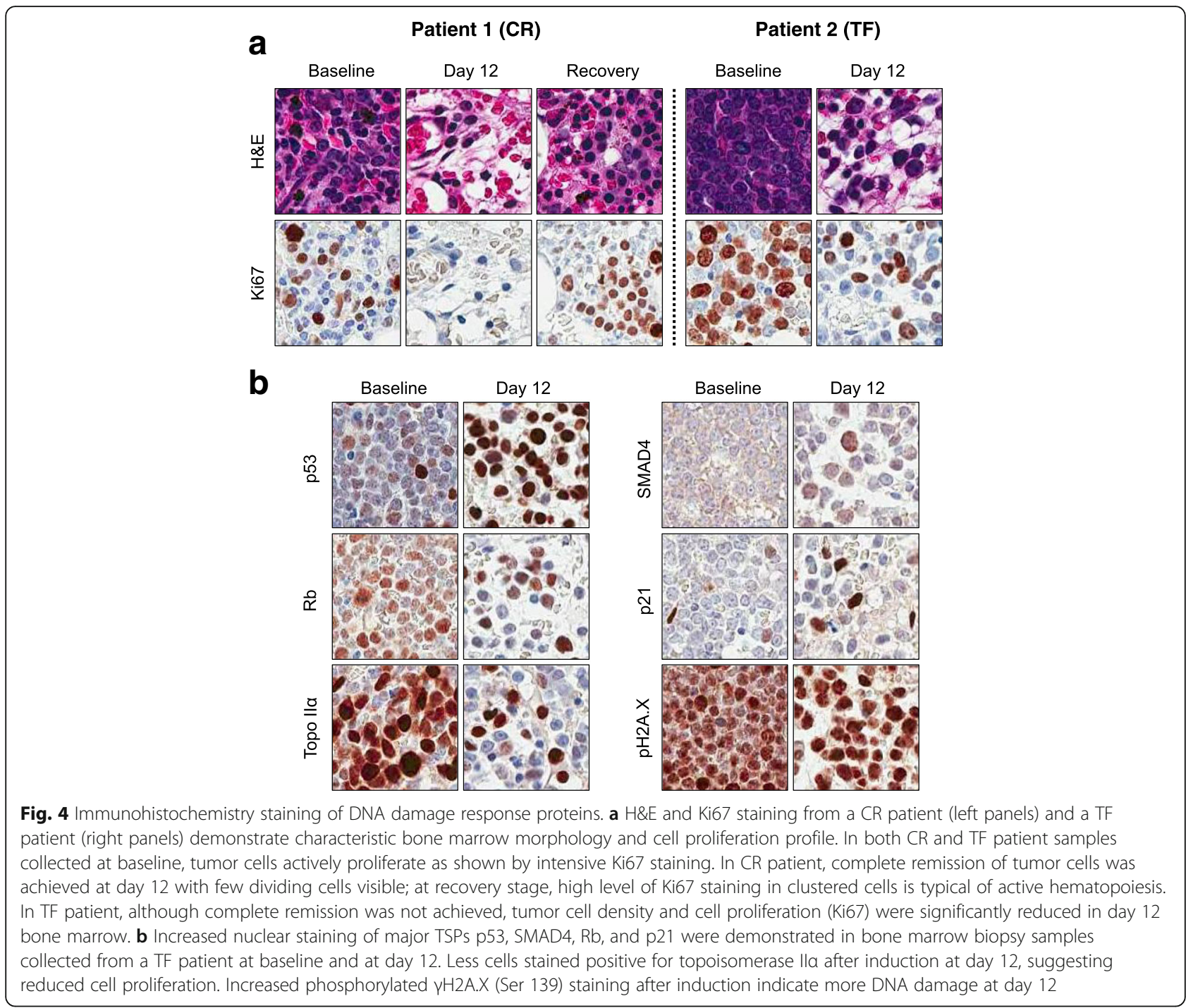

some insight into the independent contribution of selinexor to the toxicity profile. The study reported $64 \%$ of patients with neutropenic fever (vs $70 \%$ in our study), $12 \%$ with diarrhea (vs 40\%), 14\% with bacteremia (vs $25 \%$ ), and $10 \%$ with cardiac toxicity (vs 25\%), an expected toxicity in the setting of Mito exposure [30]. Additionally, more febrile neutropenia and bacteremia were observed likely due to the longer period of myelosuppression. Despite the higher number of toxicities, the majority were low grade and manageable with supportive therapies. The single case of cerebellar toxicity could be attributed to HiDAC [31, 32], as the patient was 61 years old at the time and had reduced glomerular filtration rate, yet it is worth noting that this was also observed in other phase I studies involving selinexor $[7,28]$. One patient's protracted nausea and vomiting which required TPN was assessed as selinexor-related $[12,27]$. While GI toxicities were a common reason for patient withdrawal or dose modifications in other selinexor studies [12], no patients withdrew or needed modification to the dosing during induction due to toxicities. Those who proceeded to consolidation therapy continued to tolerate the regimen, although dose reductions were needed in a couple of cases, suggesting that the 80-mg dose was less tolerable for extended longer-term use. Based on these findings, the recommended phase 2 dose of selinexor is $80 \mathrm{mg}\left(\sim 50 \mathrm{mg} / \mathrm{m}^{2}\right)$ twice a week for four doses in combination with $\mathrm{HiDAC} / \mathrm{Mito}$ for induction therapy.

The major limitations of this study were the small sample size, heterogeneous population, and short duration of follow-up that precluded drawing any definitive conclusions. Moreover, new data on a second-generation XPO1 inhibitor reported better tolerability due to decreased CNS penetration of the drug as compared to selinexor in the pre-clinical setting 
[33]. The development of a second-generation agent offers additional avenues for further study as a single agent or in combination therapy.

\section{Conclusion}

The novel class of XPO1 inhibitors have demonstrated effectiveness in disrupting cellular mechanisms that promote tumorigenesis. Selinexor is one such agent within this class that has been well studied as a single agent in AML. We demonstrated that selinexor can be feasibly combined with an existing AML regimen HiDAC/Mito, and this combination is safe with a tolerable toxicity profile. This study reported an ORR of $70 \%$ with a low induction death rate of $5 \%$. Given the potential promise of the preliminary data, further study is needed in a large phase II trial to see if these trends would continue. The RP2D of selinexor is $80 \mathrm{mg}\left(\sim 50 \mathrm{mg} / \mathrm{m}^{2}\right)$ twice a week for four doses in combination with HiDAC/Mito.

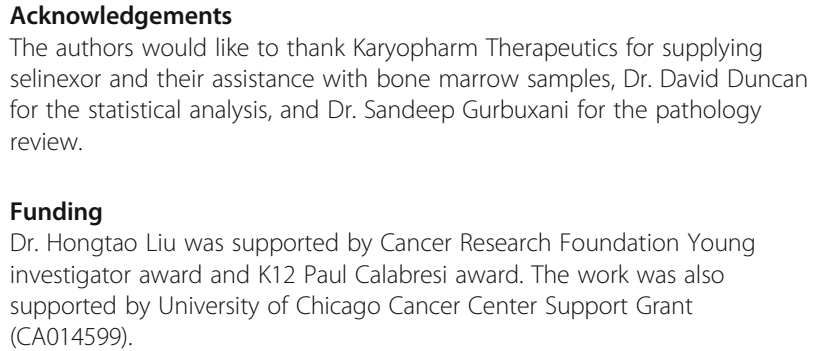

Funding

Dr. Hongtao Liu was supported by Cancer Research Foundation Young investigator award and K12 Paul Calabresi award. The work was also supported by University of Chicago Cancer Center Support Grant (CA014599).

\section{Availability of data and materials}

The dataset used during the study are available from the corresponding author on a reasonable request.

\section{Authors' contributions}

$\mathrm{HL}$ is the PI of the study, designed and wrote the protocol, and supervised the conduct of the trial. RAL, OO, and WS contributed to the design and writing of the protocol and conducted the research. AW, HW, and MG performed the research and collected and analyzed the data. HC processed the research samples and conducted the $\mathrm{IHC}$ analysis. NF processed the research samples and conducted the WT1 analysis. AA, MB, LG, MT, SK, JC, $\mathrm{EC}$, and KP conducted the research. All the authors contributed to the data analysis and the writing of the manuscript. All authors read and approved the final manuscript.

\section{Ethics approval and consent to participate}

The trial was approved by the Institutional Review Board at The University of Chicago (IRB15-0412) on 9/16/2015. All patients enrolled in the study were consented through a standardized informed consent process.

\section{Consent for publication}

Not applicable

\section{Competing interests}

Dr. Liu has research support from Karyopharm and Bristol Myers Squibb. The clinical trial was supported by Karyopharm. The co-authors reported research funding and membership on advisory boards from different companies, but reported no conflicts of interest with this study.

\section{Publisher's Note}

Springer Nature remains neutral with regard to jurisdictional claims in published maps and institutional affiliations.

\section{Author details}

'Internal Medicine/Pediatric Residency Program, University of Chicago Medicine, Chicago, IL, USA. ${ }^{2}$ Department of Medicine, Section of Hematology/Oncology, University of Chicago Medicine, 5841 S. Maryland, MC 2115, Chicago, IL 60637-1470, USA. ${ }^{3}$ Karyopharm Therapeutics Inc, 85

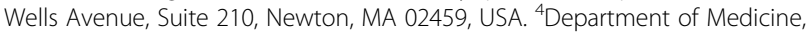
Section of Hematology/Oncology, University of Michigan Medicine, Ann Arbor, MI, USA.

Received: 25 September 2017 Accepted: 26 December 2017

Published online: 05 January 2018

\section{References}

1. Howlader N, Noone A, Krapcho M, et al. SEER cancer statistics review, 19752014. Bethesda: National Cancer Institute; 2017. https://seer.cancer.gov/csr/ 1975_2014/. Accessed 1 Jan 2017

2. Goldstone AH, Burnett AK, Wheatley $K$, Smith AG, Michael Hutchinson R, Clark RE. Attempts to improve treatment outcomes in acute myeloid leukemia (AML) in older patients: the results of the United Kingdom Medical Research Council AML11 trial. Blood. 2001;98(5):1302-11. https://doi.org/10. 1182/blood.V98.5.1302

3. Anderson JE, Kopecky KJ, Willman $\mathrm{CL}$, et al. Outcome after induction chemotherapy for older patients with acute myeloid leukemia is not improved with mitoxantrone and etoposide compared to cytarabine and daunorubicin: a Southwest Oncology Group study. Blood. 2002;100(12): 3869-76. https://doi.org/10.1182/blood-2001-12-0354.

4. Ishizawa J, Kojima K, Hail N, Tabe Y, Andreeff M. Expression, function, and targeting of the nuclear exporter chromosome region maintenance 1 (CRM1) protein. Pharmacol Ther. 2015;153:25-35. https://doi.org/10.1016/j. pharmthera.2015.06.001

5. Senapedis WT, Baloglu E, Landesman Y. Clinical translation of nuclear export inhibitors in cancer. Semin Cancer Biol. 2014;27:74-86. https://doi.org/10. 1016/.j.semcancer.2014.04.005.

6. Das A, Wei G, Parikh K, Liu D. Selective inhibitors of nuclear export (SINE) in hematological malignancies. Exp Hematol Oncol. 2015;4:7. https://doi.org/ 10.1186/s40164-015-0002-5.

7. Alexander TB, Lacayo NJ, Choi JK, Ribeiro RC, Pui CH, Rubnitz JE. Phase I study of selinexor, a selective inhibitor of nuclear export, in combination with fludarabine and cytarabine, in pediatric relapsed or refractory acute leukemia. J Clin Oncol. 2016;34(34):4094-101. https://doi.org/10.1200/JCO. 2016.67.5066.

8. Kojima K, Kornblau SM, Ruvolo V, et al. Prognostic impact and targeting of CRM1 in acute myeloid leukemia. Blood. 2013;121(20):4166-74. https://doi. org/10.1182/blood-2012-08-447581.

9. Parikh K, Cang S, Sekhri A, Liu D. Selective inhibitors of nuclear export (SINE) - a novel class of anti-cancer agents. J Hematol Oncol. 2014;7:78. https://doi.org/10.1186/s13045-014-0078-0.

10. Ranganathan $P, Y u X, N a C$, et al. Preclinical activity of a novel CRM 1 inhibitor in acute myeloid leukemia. Blood. 2012;120(9):1765-73. https://doi. org/10.1182/blood-2012-04-423160.

11. Etchin J, Montero J, Berezovskaya A, et al. Activity of a selective inhibitor of nuclear export, selinexor (KPT-330), against AML-initiating cells engrafted into immunosuppressed NSG mice. Leukemia. 2016;30(1):190-9. https://doi. org/10.1038/leu.2015.194

12. Garzon R, Savona M, Baz R, et al. A phase 1 clinical trial of single-agent selinexor in acute myeloid leukemia. Blood. 2017;129(24):3165-74. https:// doi.org/10.1182/blood-2016-11-750158.

13. Ranganathan P, Kashyap T, Yu X, et al. XPO1 inhibition using selinexor synergizes with chemotherapy in acute myeloid leukemia by targeting DNA repair and restoring topoisomerase II to the nucleus. Clin Cancer Res. 2016; 22(24):6142-52. https://doi.org/10.1158/1078-0432.CCR-15-2885.

14. Preisler HD, Venugopal $P$, Gregory SA, et al. High remission rate in acute myeloblastic leukemia with only two days of chemotherapy. Leuk Lymphoma. 2001;41(3-4):333-6. https://doi.org/10.3109/10428190109057987.

15. Devemy E, Li B, Tao M, et al. Poor prognosis acute myelogenous leukemia: 3-biological and molecular biological changes during remission induction therapy. Leuk Res. 2001;25(9):783-91. https://doi.org/10.1016/S01452126(01)00032-7.

16. Larson SM, Campbell NP, Huo D, et al. High dose cytarabine and mitoxantrone: an effective induction regimen for high-risk acute myeloid 
leukemia (AML). Leuk Lymphoma. 2012;53(3):445-50. https://doi.org/10. 3109/10428194.2011.621562.

17. Godley LA, Njiaju UO, Green M, et al. Treatment of therapy-related myeloid neoplasms with high-dose cytarabine/mitoxantrone followed by hematopoietic stem cell transplant. Leuk Lymphoma. 2010;51(6):995-1006. https://doi.org/10.3109/10428191003763468.

18. Israyelyan A, Goldstein L, Tsai W, et al. Real-time assessment of relapse risk based on the WT1 marker in acute leukemia and myelodysplastic syndrome patients after hematopoietic cell transplantation. Bone Marrow Transpl. 2015;50(1):26-33. https://doi.org/10.1038/bmt.2014.209.

19. Miyawaki S, Hatsumi N, Tamaki T, et al. Prognostic potential of detection of WT1 mRNA level in peripheral blood in adult acute myeloid leukemia. Leuk Lymphoma. 2010;51(10):1855-61. https://doi.org/10.3109/10428194.2010. 507829.

20. Le Tourneau C, Lee JJ, Siu LL. Dose escalation methods in phase I cancer clinical trials. J Natl Cancer Inst. 2009;101(10):708-20. https://doi.org/10.1093/ inci/djp079.

21. Döhner H, Estey EH, Amadori S, et al. Diagnosis and management of acute myeloid leukemia in adults: recommendations from an international expert panel, on behalf of the European LeukemiaNet. Blood. 2010;115(3):453-74. https://doi.org/10.1182/blood-2009-07.

22. Cheson BD, Bennett JM, Kopecky K, et al. Revised Recommendations of the International Working Group for diagnosis, standardization of response criteria, treatment outcomes, and reporting standards for therapeutic trials in acute myeloid leukemia. J Clin Oncol. 2003;21 (24):4642-9. https://doi.org/ $10.1200 / \mathrm{JCO} 2003.04 .036$

23. Zhang W, Ishizawa J, Mu H, Daver N, Ruvolo V. Combinatorial targeting of XPO1 and FLT3-ITD exerts synergistic anti-tumor effects in FLT3-mutated acute myeloid leukemias. Blood. 2015;126(23):Abstract 1266. http://www. bloodjournal.org/content/126/23/1266

24. Nowicki M, Ostalska-Nowicka D, Mikowiak B. Prognostic significance of Ki67negative blast cell clone in the high risk group of children treated for acute myeloid leukaemia. Folia Histochem Cytobiol. 2006;44(1):49-52.

25. Gurion R, Belnik-Plitman Y, Gafter-Gvili A, et al. Colony-stimulating factors for prevention and treatment of infectious complications in patients with acute myelogenous leukemia. In: Raanani P, editor. Cochrane database of systematic reviews. Chichester: John Wiley \& Sons, Ltd; 2012. p. CD008238. https://doi.org/10.1002/14651858.CD008238.pub3.

26. Dombret $\mathrm{H}$, Chastang $\mathrm{C}$, Fenaux $\mathrm{P}$, et al. A controlled study of recombinant human granulocyte colony-stimulating factor in elderly patients after treatment for acute myelogenous leukemia. N Engl J Med. 1995;332(25): 1678-83. https://doi.org/10.1056/NEJM199506223322504.

27. Fiedler W, Heuser M, Chromik J, et al. Phase II results of Ara-C and idarubicin in combination with the selective inhibitor of nuclear export (SINE) compound selinexor (KPT-330) in patients with relapsed or refractory AML. Blood. 2016;128(22):Abstract 341. http://www.bloodjournal.org/content/128/ 22/341. Accessed 5 Aug 2017

28. Abdul Razak AR, Mau-Soerensen M, Gabrail NY, et al. First-in-class, first-inhuman phase I study of selinexor, a selective inhibitor of nuclear export, in patients with advanced solid tumors. J Clin Oncol. 2016;34(34):4142-50. https://doi.org/10.1200/JCO.2015.65.3949.

29. Kuruvilla J, Byrd JC, Flynn JM, et al. The oral selective inhibitor of nuclear export (SINE) selinexor (KPT-330) demonstrates broad and durable clinical activity in relapsed /refractory non Hodgkin's lymphoma (NHL). Blood. 2014; 124(21):396. http://www.bloodjournal.org/content/124/21/396. Accessed 1 Feb 2017

30. Smith LA, Cornelius VR, Plummer CJ, et al. Cardiotoxicity of anthracycline agents for the treatment of cancer: systematic review and meta-analysis of randomised controlled trials. BMC Cancer. 2010;10(1):337. https://doi.org/10. 1186/1471-2407-10-337.

31. Herzig RH, Hines JD, Herzig GP, et al. Cerebellar toxicity with high-dose cytosine arabinoside. J Clin Oncol. 1987;5(6):927-32. https://doi.org/10.1200/ jco.1987.5.6.927.

32. Jolson HM, Bosco L, Bufton MG, et al. Clustering of adverse drug events: analysis of risk factors for cerebellar toxicity with high-dose cytarabine. J Natl Cancer Inst. 1992;84(7):500-5. https://doi.org/10.1093/jnci/84.7.500.

33. Etchin J, Berezovskaya A, Conway AS, et al. KPT-8602, a second-generation inhibitor of XPO1-mediated nuclear export, is well tolerated and highly active against AML blasts and leukemia-initiating cells. Leukemia. 2016; 31(April):1-8. https://doi.org/10.1038/leu.2016.145.

\section{Submit your next manuscript to BioMed Central and we will help you at every step:}

- We accept pre-submission inquiries

- Our selector tool helps you to find the most relevant journal

- We provide round the clock customer support

- Convenient online submission

- Thorough peer review

- Inclusion in PubMed and all major indexing services

- Maximum visibility for your research

Submit your manuscript at www.biomedcentral.com/submit
Biomed Central 